\title{
Applications of Computational Intelligence in Industrial and Environmental Scenarios
}

\author{
Ruggero Donida Labati, Angelo Genovese, Enrique Muñoz, \\ Vincenzo Piuri, and Fabio Scotti
}

\begin{abstract}
Computational Intelligence (CI) techniques are receiving increasing attention by the industrial and academic communities involved in the design of automatic systems for industrial and environmental monitoring and control applications. CI techniques are able to aggregate inputs from several heterogeneous sensors, adapt themselves to wide ranges of operational and environmental conditions, and cope with incomplete or noise-affected data. With current computing architectures evolving towards smaller size, higher computational power, and more affordable cost, a great number of devices can embed CI techniques to support different kinds of applications. In this paper, we present a survey of the recent CI methods designed for the main processing steps of industrial and environmental monitoring systems.
\end{abstract}

\section{Introduction}

In industrial and environmental monitoring and control applications, experts in the field usually design physical models and perform a statistical analysis to infer knowledge about the observed phenomena. With such knowledge, it is possible to design and implement the corresponding monitoring and control methods. However, taking into account all the variables of the observed phenomenon can make the modeling and design process complex, with the result of obtaining inaccurate systems. Variations in the observed processes may also occur (e.g., wearing of the industrial machinery during the monitoring of industrial processes and meteorological anomalies while performing environmental monitoring), introducing the need of manual and complex adjustments to the models, or even making the system inadequate. Monitoring and control systems for industrial and environmental applications are frequently implemented in non-ideal operational conditions, which introduce noise

Department of Computer Science - Università degli Studi di Milano via Bramante 65, I-26013 Crema, Italy. e-mail: \{firstname.lastname\}@unimi.it 
in the signals acquired by the sensors and increase the complexity of the designed model [29].

Computational Intelligence (CI) techniques permit to design and implement intelligent systems for industrial and environmental monitoring and control applications that $i$ ) do not require any modeling of the process based on expert knowledge and ii) can infer a model by learning from examples. The CI methods most commonly used are Artificial Neural Networks (ANN) (such as, feed-forward networks, radial basis function networks, self-organizing maps, and convolutional neural networks), Support Vector Machines (SVM), Fuzzy Logic, and Evolutionary Computation (EC) [54].

These techniques can dynamically update the parameters of the model as the system is running, for instance by providing more recent examples from which the model can be updated. Intelligent monitoring systems have also the advantage of increasing the robustness to noisy data with respect to traditional approaches. The constant increase of computational power of processing architectures, along with the reduced size and cost of the devices, permits to use a higher number of sensors $[48,70]$ and therefore a more widespread diffusion of CI-based intelligent monitoring systems for a greater number of applications [22,113]. Examples of industrial applications include production monitoring [10], control of robots [77], detection of faults in the machinery [52,84], and control of the quality of industrial processes [9]. In environmental scenarios, applications include forecasting [51,73] and detection of hazardous situations (e.g., oil spill [92]).

Intelligent monitoring and control systems based on CI techniques share a similar architecture that comprises the following steps: $i$ ) data acquisition, $i i$ ) data preprocessing, iii) feature extraction and selection, $i v$ ) data fusion, $v$ ) classification, regression, or clustering, $v i$ ) system optimization and evaluation. Studies in the literature proposed applications of CI techniques for each of these steps [9, 13, 72]. Fig. 1 illustrates the steps of the intelligent monitoring and control systems, together with the main applications of CI techniques.

This paper presents an overview on CI techniques for the design of intelligent monitoring systems in industrial and environmental scenarios. The paper is organized in as follows. Section 2 overviews the main CI techniques proposed in the literature. Section 3 presents applications of industrial and environmental monitoring. Section 4 describes the architecture of intelligent monitoring and control systems, by overviewing the methods in the literature using CI techniques for each step of the systems. Section 5 concludes the paper.

\section{Overview of Computational Intelligence techniques}

CI techniques are methods that enable intelligent behavior in monitoring and control systems, with the features of being flexible and adaptive [35]. Among the advantages of CI techniques, there are the capabilities of working with noisy or incomplete data, providing approximate solutions, adapting to changes in operational and environ- 


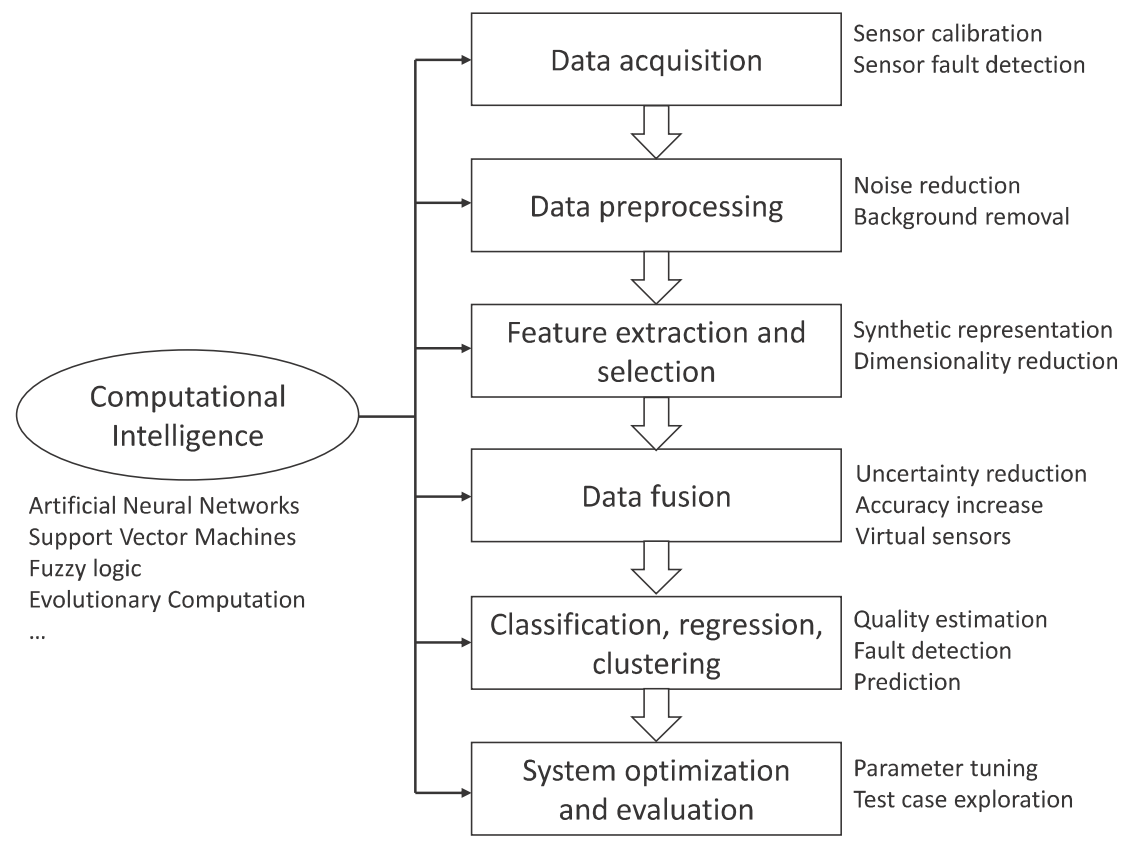

Fig. 1 Outline of an intelligent monitoring system for industrial and environmental applications. The figure enumerates the main applications of CI techniques for each step of monitoring systems

mental conditions, and featuring a low computational complexity. These advantages have altogether contributed to the increasing use of CI techniques for industrial and environmental applications $[9,61]$.

This section presents the CI techniques mostly used in the field of industrial and environmental monitoring, such as Artificial Neural Networks (ANN), Support Vector Machines (SVM), Fuzzy Logic, and Evolutionary Computation (EC) [54].

ANNs exploit several layers of interconnected neurons to reproduce and mimic the reasoning ability of the human brain [8]. A learning procedure permits to adjust the weights associated to each neuron and the corresponding transfer functions. In this manner, the ANN can adapt itself to the training examples and process data to achieve approximate solutions with fault tolerance capabilities and low computational complexity. Several topologies of ANNs appear in the literature, presenting differences in the structure of the network and in the interconnections between the neurons. The topologies mostly used are feed-forward neural networks, radial basis function networks, and self-organizing maps [36,49]. Recently, convolutional neural networks and other deep learning approaches have also been attracting the attention of the academic and industrial communities [64].

SVMs are a particular type of kernel-based methods that have the purpose of projecting the input data onto a space with a higher dimensionality to facilitate the learning process. In particular, the learning process in SVMs optimizes a convex sur- 
face without ending in local optima. One of the main advantages of SVMs consists in having a limited number of parameters, resulting in a faster tuning process [19].

Methods based on Fuzzy Logic have the peculiarity of dealing with information expressed with various degrees of uncertainty. For this reason, they are especially well suited to deal with incomplete or imprecise data. Advantages of Fuzzy systems include the use of linguistic concepts and the capability to deal with conflicting objectives by designing knowledge bases [97].

Methods using EC perform a global optimization by mimicking the biological evolution. In particular, the algorithms start with an initial population of solutions that iteratively interact among themselves by following principles derived from natural evolution, to improve their overall quality. The process of creating the initial population involves the assignment of random values to the parameters, or the use of heuristics tailored for the application scenario. Methods for updating the population include random permutations, crossover, or selection. Several algorithms based on EC have been proposed in the literature, including genetic algorithms and swarm intelligence (e.g., ant colony optimization and particle swarm optimization) [91].

\section{Applications of industrial and environmental monitoring}

Hardware processing architectures nowadays feature increased computing power, reduced size, and limited cost. For example, current smartphones and mini-PCs can easily exceed the computational capacity of desktop workstations made few years ago [82]. This increased availability of powerful and low-cost architectures allowed a greater number of monitoring infrastructures to be deployed in several novel scenarios [22,113]. Furthemore, advances in the manufacturing of CMOS sensors have enabled the use of long-range vision systems using off-the-shelf equipment $[20,111]$. Current devices have high computational power, extended battery life, and advanced networking capabilities [42], which are key factors to enable the deployment of sensors for intelligent monitoring in novel scenarios such as industrial and environmental applications, where resilience and robustness are of paramount importance. Therefore, the research is moving towards a greater use of CI techniques, due to their capability to aggregate inputs from different sensors and learn the relationship between the sensed data and the parameters of the observed phenomenon. At the same time, CI methods can adapt to variations in operational conditions. Thanks to these characteristics, CI techniques permit an accurate, robust, and fast detection of anomalies in industrial and environmental monitoring applications $[9,37,61]$. 


\subsection{Industrial monitoring}

Intelligent monitoring systems can be classified according to their architecture in two main categories: $i$ ) centralized systems and ii) distributed systems.

Centralized monitoring systems typically employ vision-based sensors (e.g., CMOS cameras) to sense data at long distances. Vision-based systems are used since they can perform a touchless and non-destructive monitoring of the industrial process [90]. A single pan-and-tilt camera could cover a large area [46], thus reducing the need of having multiple sensing nodes. Moreover, it is possible to extract multiple features from the images captured, further reducing the need and cost of deploying multiple sensors. For instance, vision-based systems can monitor volume [30], surface defects [44,83], or analyze the particle size distribution $[27,31,38]$ of raw materials used in manufacturing processes.

Distributed monitoring systems often use Wireless Sensor Networks (WSNs) to build low-cost, robust, and pervasive monitoring networks composed by several inexpensive sensor nodes. The use of multiple and redundant sensor nodes allows the WSN to reconfigure itself in cases of changes of the physical placement of the nodes or sensor failures $[48,52]$.

CI techniques have widely shown their suitability to perform monitoring tasks in industrial environments. Examples of their applications include the detection of anomalies in tools and machinery [14, 52, 53, 84, 103], analysis of quantities of interest of raw materials or final products (e.g., shape, volume, or weight) $[27,30,38]$, analysis of the quality of the manufactured product $[4,7,28,40,44,83,85,86]$.

\subsection{Environmental monitoring}

Environmental monitoring systems can be classified according to their architecture into three types [10,22,23]: $i$ ) centralized systems, ii) distributed systems, and iii) remote sensing systems.

Centralized systems typically employ a single vision-based sensing node, to provide long-range detection capabilities in situations that require to monitor wide environments. Example of centralized environmental monitoring systems are wildfire detection technologies based on a low-cost camera [32,47].

Distributed systems often use WSNs since large-scale sensor networks composed by small and inexpensive nodes can be deployed easily in areas difficult to reach (e.g., volcanos and oceans) or on wide environments (e.g., mountain range). The advanced networking capabilities of the nodes and a battery life sufficient to carry out the monitoring task for long time periods allow the sensor nodes to self-organize even if they are deployed automatically from a distance, for example, when launched from an airplane $[61,70]$. Environmental monitoring systems using a distributed architecture can monitor climate change [68], structural health [106], water quality $[78,79,105]$, or meteorology [51]. 
Remote sensing systems acquire data using satellite imagery to monitor environmental phenomena on a planetary scale. Examples of remote sensing systems for environmental monitoring include methods for detecting seismic activity [107], air pollution [110], and water pollution [92]. Satellite imagery can also detect meteorological phenomena and perform weather forecasts [73].

CI techniques are often used for environmental monitoring. They are widely used to classify features extracted from sensed data [32,73, 92, 107, 110], perform forecasts from time series [51,79], and aggregate data obtained from heterogeneous sensors (e.g., humidity, vibrations, temperature, chemical concentrations) in WSNs $[61,68,78,106]$.

\section{Architecture of intelligent monitoring and control systems}

The structure of industrial and environmental monitoring systems typically includes six main steps [9], as illustrated in Fig. 1. CI techniques can be used to perform or enhance each step of the system. In the following, we overview the applications of CI for each single step:

1. Data acquisition: the monitoring system usually collects one-dimensional signals, two-dimensional signals, three-dimensional models, or frame sequences. In this step, CI techniques can perform data linearization, sensor calibration, and system diagnosis.

2. Data preprocessing: the noise in the collected data is reduced, and the pattern of interest is extracted from the input multi-dimensional signals (e.g., image segmentation).

3. Feature extraction and selection: the features of interest for the monitoring application are extracted from the enhanced data. In this step, CI techniques can be used to obtain a synthetic representation of the phenomenon and reduce the dimensionality of the extracted features.

4. Data fusion: the system aggregates data or features coming from different sources, obtaining a compact description of the phenomenon of interest. In this step, CI techniques can increase the accuracy of traditional methods and reduce the uncertainty of virtual measurements.

5. Classification, regression or clustering: the monitoring system analyzes the features to derive patterns of interest for the monitored phenomenon. In this step, CI techniques can be used to perform fault detection, quality estimation, or prediction.

6. System optimization and evaluation: the parameters of the monitoring system are adjusted to improve its performance. In this step, CI techniques can be used to tune the parameters of the system, modify the used model according to new operational conditions, or evaluate the performance of the system by exploring different test cases. 


\begin{tabular}{|c|c|c|c|c|c|c|c|c|}
\hline \multirow{3}{*}{ Step } & \multirow{3}{*}{ Application } & \multicolumn{7}{|c|}{ CI technique } \\
\hline & & \multicolumn{4}{|c|}{ ANN } & \multirow{2}{*}{ SVM } & \multirow{2}{*}{ Fuzzy } & \multirow{2}{*}{ EC } \\
\hline & & FF & RBF & SOM & CNN & & & \\
\hline \multirow{2}{*}{$\begin{array}{l}\text { Data } \\
\text { acquisition }\end{array}$} & $\begin{array}{l}\text { Sensor } \\
\text { calibration }\end{array}$ & [24] [57] & - & - & - & [74] & - & - \\
\hline & $\begin{array}{l}\text { Sensor fault } \\
\text { detection }\end{array}$ & - & - & - & - & - & [75] & [89] \\
\hline \multirow{2}{*}{$\begin{array}{l}\text { Data } \\
\text { preprocessing }\end{array}$} & $\begin{array}{l}\text { Noise } \\
\text { reduction }\end{array}$ & $\begin{array}{c}{[5][6]} \\
{[17][98]}\end{array}$ & - & - & - & {$[102]$} & [96] & {$[81]$} \\
\hline & $\begin{array}{l}\text { Background } \\
\text { removal }\end{array}$ & {$[88]$} & - & - & - & - & [2] [27] & - \\
\hline \multirow{2}{*}{$\begin{array}{l}\text { Feature } \\
\text { extraction } \\
\text { and selection }\end{array}$} & $\begin{array}{l}\text { Synthetic } \\
\text { representation }\end{array}$ & $\begin{array}{c}{[43][65]} \\
{[116]}\end{array}$ & - & $\begin{array}{c}{[67][87]} \\
{[104]}\end{array}$ & [53] [115] & - & - & \\
\hline & $\begin{array}{l}\text { Dimensionality } \\
\text { reduction }\end{array}$ & - & - & - & - & - & - & $\begin{array}{c}\text { [58] [108] } \\
{[109]}\end{array}$ \\
\hline \multirow{3}{*}{ Data fusion } & $\begin{array}{l}\text { Uncertainty } \\
\text { reduction }\end{array}$ & {$[69]$} & - & {$[63]$} & - & {$[69]$} & $\begin{array}{c}{[50][59]} \\
{[93]}\end{array}$ & - \\
\hline & Accuracy increase & [95] & - & - & - & {$[14]$} & {$[103]$} & - \\
\hline & Virtual sensors & [71] & - & - & - & - & {$[55]$} & - \\
\hline \multirow{3}{*}{$\begin{array}{l}\text { Classification, } \\
\text { regression, } \\
\text { clustering }\end{array}$} & Quality estimation & [7] [28] & - & - & - & [85] & {$[60]$} & {$[60]$} \\
\hline & Fault detection & [103] & - & - & {$[66]$} & {$[14]$} & {$[103]$} & - \\
\hline & Prediction & $\begin{array}{c}{[12][16]} \\
{[32][79]} \\
{[92][107]}\end{array}$ & {$[3]$} & - & [11] [25] & $\begin{array}{c}{[45][62]} \\
{[105]}\end{array}$ & $\begin{array}{c}{[33][55]} \\
{[76][114]}\end{array}$ & {$[105]$} \\
\hline \multirow{2}{*}{$\begin{array}{l}\text { System } \\
\text { optimization } \\
\text { and testing }\end{array}$} & $\begin{array}{l}\text { Parameter } \\
\text { tuning }\end{array}$ & - & - & - & - & - & - & $\begin{array}{c}{[1][18]} \\
{[112]}\end{array}$ \\
\hline & $\begin{array}{l}\text { Test case } \\
\text { exploration }\end{array}$ & - & - & - & - & - & - & $\begin{array}{c}{[94][99]} \\
{[100]}\end{array}$ \\
\hline
\end{tabular}

Note. ANN: Artificial Neural Network; FF: Feed-Forward Neural Network; RBF: Radial Basis Function Neural Network; SOM: Self-Organizing Maps; CNN: Convolutional Neural Network; SVM: Support Vector Machine; EC: Evolutionary Computation.

Table 1 Summary of the different CI techniques used for each step of monitoring systems and the corresponding industrial and environmental applications

Table 1 summarizes the different $\mathrm{CI}$ techniques that have been proposed for each step performed by the monitoring systems and the corresponding industrial and environmental applications. In the remainder of this section, we elaborate more on the use of CI techniques in the different steps.

\subsection{Data acquisition}

The data acquisition step collects information in the form of one-dimensional (e.g., time series) or multi-dimensional signals (e.g., images, three-dimensional models, frame sequences). To collect data, industrial and environmental monitoring systems can use sensors mimicking the human senses and sensors collecting physical quan- 
tities, including pressure, humidity, temperature, acceleration, or presence of chemicals. CI techniques can be used to enhance the data acquisition step by performing sensor calibration $[24,57,74,89]$ and sensor fault detection $[37,75,80]$. CI techniques widely used in this contex are ANNs [24,57], SVMs [74], Fuzzy Logic [75], and EC [89].

To achieve a more pervasive monitoring, the extracted information can be exchanged using cloud-based infrastructure and internet of things. We note, however, that they can introduce security and privacy concerns, especially regarding industrial trade secrets [21].

\subsection{Data preprocessing}

Data acquired in industrial and environmental scenarios can be affected by noise introduced by different non-ideal conditions, such as adverse operational conditions, variations in the environmental parameters, and interferences in the communication channels. The preprocessing step has then the purpose of enhancing the quality of the acquired data, remove the noise in the signals, and extract the pattern of interest.

Typically, ad-hoc filters process one-dimensional or multi-dimensional signals to enhance the quality of the data according to the characteristics of the monitoring application, by removing frequencies that do not represent the information to be analyzed or by restoring the original signal from the acquired noisy data. CI techniques are also adopted for data filtering, using methods based on ANNs $[5,6,17,98]$, SVMs [102], Fuzzy Logic [96], and EC [81].

In systems based on multi-dimensional signals (e.g., images or frame sequences) it is particularly important to segment the regions of interest from the background, so that the subsequent processing steps can avoid to consider unnecessary information [54]. To this purpose, CI techniques can be applied to train a model able to recognize the foreground from the background by learning from examples. In particular, ANNs have been used to extract regions of interest of bee foraging using images acquired from satellites [88], and methods based on Fuzzy Logic have been applied to select wood particles for panel production [27] or periods of interest from time-series of chemical processes [2].

\subsection{Feature extraction and selection}

Data captured by the sensors have usually a high dimensionality (e.g., acquisitions of long time series or large images from a high number of sensors) and cannot be directly processed to accurately infer the model of the observed phenomena. The feature extraction and selection steps have then the purpose of reducing the dimensionality of the preprocessed data to obtain a synthetic representation, usually called a feature vector [54]. Feature extraction and selection methods based on CI techniques 
permit to significantly reduce the amount of data to be processed by the subsequent steps of the systems, thus reducing the probability of overfitting problems and the need of computational resources [26]. Feature extraction and feature selection are typically performed in sequence. First, feature extraction methods compute a feature set with lower size with respect to the input multidimensional signal (e.g., the entire size of the captured image). Second, feature selection methods further reduce the dimensionality of the feature space by selecting a subset of the most discriminative features.

Traditional techniques for feature extraction include the linear discriminant analysis, principal component analysis, independent component analysis, polynomial approximation, and multidimensional scaling [54]. In industrial and environmental monitoring, examples of feature vectors include abstract representations of the gray-level variations that describe irregularities of a surface [34], the shape of a moving region [32], and anomalies in frequency ranges of the input signal [28, 72].

CI techniques based on ANNs have been widely used to perform feature extraction in industrial and environmental monitoring applications, with methods using feed-forward neural networks [43, 65, 116], self-organizing maps [67, 87, 104], radial basis function networks [15], and convolutional neural networks [53, 115].

Feature selection techniques search for the subset of features that best describes the observed phenomenon, by using strategies more efficient with respect to the exhaustive search, which can be unfeasible in terms of computational complexity in the case of feature vectors with high dimensionality. Traditional techniques are frequently based on Sequential Floating Search or Sequential Forward (Backward) Selection [54]. CI techniques can also perform feature selection: in particular, methods based on EC are especially well suited for this purpose [58, 108, 109].

\subsection{Data fusion}

In monitoring applications, the data fusion step can reduce uncertainty in the collected data, for instance, by aggregating information obtained from several sensors of the same type [56], and reducing possible problems due to conflicting data and outliers. Data fusion techniques can increase the accuracy of the monitoring process by fusing information of heterogeneous sensors to achieve a more comprehensive description of the phenomenon [39]. Data fusion methods can also create virtual (or soft) sensors to indirectly measure physical quantities by inferring knowledge from the fusion of other information [41].

Fusion methods for uncertainty reduction are especially used in WSNs to aggregate the data collected by the heterogeneous sensors. CI techniques are especially suited to combine numerous sources of information to derive knowledge and perform an intelligent monitoring [61]. In industrial monitoring applications, Fuzzy Logic has been used to detect faults [59]. In environmental monitoring scenarios, self-organizing maps have been used to derive information about flood levels [63] and Fuzzy Logic has been used to monitor the temperature [93]. 
In industrial and environmental applications, several CI techniques have been used to increase the accuracy of the monitoring process. In particular, methods based on ANNs are widely used in industrial scenarios to combine the information obtained by different sensors. For example, ANNs have been used with the purpose of detecting the wearing of the tools used in the production machinery [95]. Similarly, methods based on SVM have been used to determine faults in the motors [14]. A combination of ANNs and Fuzzy Logic has also been used to model and monitor anomalies in hybrid energy systems [103]. In environmental monitoring, methods for aggregating data to reduce uncertainty in detecting floods have applied both ANNs and SVMs [69]. Techniques based on Fuzzy Logic have been used to detect landslides by aggregating geophysical data [50].

CI techniques have also been applied for the creation of virtual sensors for the indirect monitoring of physical quantities. When measuring the desired physical quantity is too complex or expensive, CI techniques can be applied to infer knowledge about the desired physical quantity, for example, by aggregating information acquired by different sources. In industrial applications, virtual sensors based on a Neuro-Fuzzy system have been proposed to monitor a hydrogen electrolyzer [55], while in environmental scenarios ANNs have been used to perform the localization of odor sources [71].

\subsection{Classification, regression and clustering}

The classification, regression, and clustering steps have the purpose of creating a model of the industrial or environmental phenomenon under analysis. These steps process the extracted features by associating to each observation a discrete label (classification) or a continuous value (regression), or by grouping them according to certain distance measures (clustering). CI techniques have extensively demonstrated their suitability for these tasks, since they can obtain flexible and adaptive models of the phenomenon through training and are robust to noisy or incomplete data. CI methods can approximate a model by using a finite number of examples, provide solutions by using a limited amount of resources, and mimic the human generalization ability $[8,49]$.

In classification, a discrete label is assigned to sets of features sharing some characteristics that differentiate them from the rest of the samples [101] (e.g., lowquality products, machinery faults, and meteorological anomalies). In industrial monitoring applications, CI techniques can detect low-quality outcomes of the production processes. The techniques mostly used are ANNs [7, 28], SVMs [85], and Evolutionary-Fuzzy systems [60]. CI methods can also perform fault detection in industrial scenarios, using convolutional neural networks [66], Neuro-Fuzzy systems [103] or SVMs [14]. As for environmental monitoring applications, ANNs can predict natural disasters [32,107] and pollution [92]. Recent applications based on deep learning techniques have also addressed the problem of disaster prediction $[11,25]$. 
In regression tasks, the goal is to estimate a continuous value from the set of features associated to each sample. In industrial monitoring applications, CI techniques often use regression to predict energy levels or quality parameters. In particular, ANNs can predict energy consumption [12] and Fuzzy Logic can estimate quality parameters in industrial processes [114]. Fuzzy Logic [55] and radial basis function networks [3] using regression-based learning procedures have also been proposed to create virtual sensors for industrial monitoring. As for environmental monitoring applications, regression-based techniques can predict meteorological phenomena or pollution levels, with ANNs used to forecast the water quality in rivers [79] and a combination of SVMs and EC used to predict air quality in urban areas [45, 105].

In clustering, the objective is to group samples according to certain distance measures. Differently from classification techniques, clustering algorithms typically use unsupervised learning procedures that do not require any a-priori knowledge on the semantics of the groups of similar data present in the considered set of samples. In industrial monitoring applications, CI techniques using clustering procedures based on Fuzzy Logic have been used to group the energy demands in different periods of time for prediction purposes [76]. Techniques based on SVMs have also been used to predict the optimal maintenance scheduling [62]. In environmental monitoring applications, clustering techniques based on ANNs have been proposed to predict seismic activities [16] and Fuzzy clustering methods have been used to estimate pollution [33].

\subsection{System optimization and evaluation}

Monitoring and control systems for industrial and environmental applications may include many models and a huge amount of parameters. Tuning these parameters can became a very difficult process, because the variables can interact in unpredictable ways. Therefore, tuning the systems based on the exhaustive exploration of all the possible combinations of parameters is almost impossible. Other simple techniques, such as trial and error, can only find sub-optimal configurations [9]. Differently, CI techniques based on EC, such as tabu search or ant colony optimization, represent effective strategies to find near-optimal solutions [18]. For instance, advanced EC techniques have been used to optimize the parameters of milling machines [112] and multi-generation energy systems [1].

Another important task to optimize the behavior of industrial and environmental monitoring and control systems consists in testing their modules. This task permits to avoid expensive errors and improve the overall performance of the system. In most cases, this task cannot be performed only by human operators because of the large domain of potential test cases, frequently too complex to be explored exhaustively. Moreover, testing procedures performed only by human operators can increase the cost of the systems, because they could miss important errors.

EC-based techniques have emerged as a viable solution to automate the testing process. This kind of techniques can generate effective test cases [100], and have 
been successfully applied to complex control systems, such as railway automation [94] and car production [99].

\section{Conclusions}

The design of industrial and environmental monitoring systems is a complex task because it has to face intricate processes, complex phenomena, noisy and missing data. Methods based on mathematical models or statistical analysis require careful engineering, and in many cases obtain incomplete or inaccurate models. In addition, such methods are difficult to apply when the data are noisy or incomplete. In contrast, Computational Intelligence (CI) methods such as Artificial Neural Networks, Support Vector Machines, Fuzzy Logic, or Evolutionary Computation can obtain flexible models that can cope with noise, data incompleteness, and varying operational conditions, while having a limited computational complexity.

This work has reviewed recent advances in the application of CI techniques to industrial and environmental monitoring scenarios. We have provided a taxonomy of the methods by grouping them according to the step of the design process that they cover. In particular, we considered the following steps: data acquisition, data preprocessing, feature extraction, data fusion, classification, regression, clustering and system optimization. In this way, we provided a complete design methodology. We have also illustrated the suitability of CI methods to face industrial and environmental monitoring and control problems. In particular, we have shown that CI approaches can obtain better performance compared with traditional methods and can provide adaptable and robust solutions. Recent advances in CI, such as deep learning and hybrid systems, make us believe that the applications of CI to industrial and environmental problems will continue to grow, with further improvements in terms of adaptability and performance.

Acknowledgements This work was supported in part by the EC within the $\mathrm{H} 2020$ program under grant agreement 644597 (ESCUDO-CLOUD).

\section{References}

1. Ahmadi, P., Dincer, I., Rosen, M.A.: Thermodynamic modeling and multi-objective evolutionary-based optimization of a new multigeneration energy system. Energy Conversion and Management 76, 282-300 (2013)

2. Alaei, H.K., Salahshoor, K., Alaei, H.K.: A new integrated on-line fuzzy clustering and segmentation methodology with adaptive PCA approach for process monitoring and fault detection and diagnosis. Soft Computing 17(3), 345-362 (2013)

3. Alexandridis, A.: Evolving RBF neural networks for adaptive soft-sensor design. Int. Journal of Neural Systems 23(6), 1350,029 (2013) 
4. Alippi, C., Braione, P.: Classification methods and inductive learning rules: what we may learn from theory. IEEE Trans. on Systems, Man, and Cybernetics, Part C (Applications and Reviews) 36(5), 649-655 (2006)

5. Alippi, C., Casagrande, E., Fumagalli, M., Scotti, F., Piuri, V., Valsecchi, L.: An embedded system methodology for real-time analysis of railways track profile. In: Proc. of the $19^{\text {th }}$ IEEE Instrumentation and Measurement Technology Conf. (IMTC), pp. 747-751 (2002)

6. Alippi, C., Casagrande, E., Scotti, F., Piuri, V.: Composite real-time image processing for railways track profile measurement. IEEE Trans. on Instrumentation and Measurement 49(3), 559-564 (2000)

7. Alippi, C., D’Angelo, G., Matteucci, M., Pasquettaz, G., Piuri, V., Scotti, F.: Composite techniques for quality analysis in automotive laser welding. In: Proc. of the 2003 IEEE Int. Symp. on Computational Intelligence for Measurement Systems and Applications (CIMSA), pp. 72-77 (2003)

8. Alippi, C., Ferrero, A., Piuri, V.: Artificial intelligence for instruments and measurement applications. IEEE Instrumentation Measurement Magazine 1(2), 9-17 (1998)

9. Alippi, C., Roveri, M., Piuri, V., Scotti, F.: Computational intelligence in industrial quality control. In: Proc. of the 2005 IEEE Int. Workshop on Intelligent Signal Processing (WISP), pp. 4-9. Faro, Portugal (2005)

10. Amigoni, F., Brandolini, A., Caglioti, V., Di Lecce, V., Guerriero, A., Lazzaroni, M., Lombardi, F., Ottoboni, R., Pasero, E., Piuri, V., Scotti, O., Somenzi, D.: Agencies for perception in environmental monitoring. IEEE Trans. on Instrumentation and Measurement 55(4), 1038-1050 (2006)

11. Amit, S.N.K.B., Shiraishi, S., Inoshita, T., Aoki, Y.: Analysis of satellite images for disaster detection. In: Proc. of the 2016 IEEE Int. Geoscience and Remote Sensing Symp. (IGARSS), pp. 5189-5192 (2016)

12. Azadeh, A., Ghaderi, S.F., Sohrabkhani, S.: Annual electricity consumption forecasting by neural network in high energy consuming industrial sectors. Energy Conversion and Management 49(8), 2272-2278 (2008)

13. Azar, T.A., Vaidyanathan, S.: Computational intelligence applications in modeling and control. Springer Int. Publishing (2015)

14. Banerjee, T.P., Das, S.: Multi-sensor data fusion using support vector machine for motor fault detection. Information Sciences 217, 96-107 (2012)

15. Bellocchio, F., Borghese, N.A., Ferrari, S., Piuri, V.: 3D surface reconstruction: multi-scale hierarchical approaches. Springer (2013)

16. Braeuer, B., Bauer, K.: A new interpretation of seismic tomography in the southern Dead Sea basin using neural network clustering techniques: Interpretation of tomography in the SDSB. Geophysical Research Letters 42(22), 9772-9780 (2015)

17. Burger, H.C., Schuler, C.J., Harmeling, S.: Image denoising: Can plain neural networks compete with BM3D? In: Proc. of the 2012 IEEE Conf. on Computer Vision and Pattern Recognition (CVPR), pp. 2392-2399 (2012)

18. Cadenas, J.M., Garrido, M.C., Muñoz, E.: Facing dynamic optimization using a cooperative metaheuristic configured via fuzzy logic and SVMs. Applied Soft Computing 11(8), 5639$5651(2011)$

19. Campbell, C.: An introduction to kernel methods. In: R.J. Howlett, L.C. Jain (eds.) Radial basis function networks: design and applications. Springer Verlag, Berlin (2000)

20. Charfi, Y., Wakamiya, N., Murata, M.: Challenging issues in visual sensor networks. IEEE Wireless Communications 16(2), 44-49 (2009)

21. De Capitani di Vimercati, S., Foresti, S., Livraga, G., Samarati, P.: Data privacy: Definitions and techniques. Int. Journal of Uncertainty, Fuzziness and Knowledge-Based Systems 20(6), 793-817 (2012)

22. De Capitani di Vimercati, S., Genovese, A., Livraga, G., Piuri, V., Scotti, F.: Privacy and security in environmental monitoring systems: Issues and solutions. In: J.R. Vacca (ed.) Computer and Information Security Handbook, $2^{\text {nd }}$ edn., pp. 835-853. Morgan Kaufmann, Boston (2013) 
23. De Capitani di Vimercati, S., Livraga, G., Piuri, V., Scotti, F.: Privacy and security in environmental monitoring systems. In: Proc. of the 2012 IEEE $1^{\text {st }}$ AESS European Conf. on Satellite Telecommunications (ESTEL), pp. 1-6 (2012)

24. Di Natale, C., Davide, F.A.M., D’Amico, A., Göpel, W., Weimar, U.: Sensor arrays calibration with enhanced neural networks. Sensors and Actuators B: Chemical 19(1), 654-657 (1994)

25. Ding, A., Zhang, Q., Zhou, X., Dai, B.: Automatic recognition of landslide based on CNN and texture change detection. In: Proc. of the $201631^{\text {st }}$ Youth Academic Annual Conf. of Chinese Association of Automation (YAC), pp. 444448 (2016)

26. Domingos, P.: A few useful things to know about machine learning. Communications of the ACM 55(10), 78-87 (2012)

27. Donida Labati, R., Genovese, A., Muñoz, E., Piuri, V., Scotti, F., Sforza, G.: Improving OSB wood panel production by vision-based systems for granulometric estimation. In: Proc. of the 2015 IEEE $1^{\text {st }}$ Int. Forum on Research and Technologies for Society and Industry (RTSI), pp. 557-562 (2015)

28. Donida Labati, R., Genovese, A., Muñoz, E., Piuri, V., Scotti, F., Sforza, G.: Analyzing images in frequency domain to estimate the quality of wood particles in OSB production. In: Proc. of the 2016 IEEE Int. Conf. on Computational Intelligence and Virtual Environments for Measurement Systems and Applications (CIVEMSA). Budapest, Hungary (2016)

29. Donida Labati, R., Genovese, A., Muñoz, E., Piuri, V., Scotti, F., Sforza, G.: Computational intelligence for industrial and environmental applications. In: Proc. of the 2016 IEEE $8^{\text {th }}$ Int. Conf. on Intelligent Systems (IS), pp. 8-14 (2016)

30. Donida Labati, R., Genovese, A., Piuri, V., Scotti, F.: Low-cost volume estimation by twoview acquisitions: A computational intelligence approach. In: Proc. of the 2012 Int. Joint Conf. on Neural Networks (IJCNN), pp. 1-8 (2012)

31. Donida Labati, R., Genovese, A., Piuri, V., Scotti, F.: A virtual environment for the simulation of $3 \mathrm{D}$ wood strands in multiple view systems for the particle size measurements. In: Proc. of the 2013 IEEE Int. Conf. on Computational Intelligence and Virtual Environments for Measurement Systems and Applications (CIVEMSA), pp. 162-167 (2013)

32. Donida Labati, R., Genovese, A., Piuri, V., Scotti, F.: Wildfire smoke detection using computational intelligence techniques enhanced with synthetic smoke plume generation. IEEE Trans. on Systems, Man and Cybernetics: Systems 43(4), 1003-1012 (2013)

33. D’Urso, P., Di Lallo, D., Maharaj, E.A.: Autoregressive model-based fuzzy clustering and its application for detecting information redundancy in air pollution monitoring networks. Soft Computing 17(1), 83-131 (2013)

34. Dutta, S., Datta, A., Chakladar, N.D., Pal, S.K., Mukhopadhyay, S., Sen, R.: Detection of tool condition from the turned surface images using an accurate grey level co-occurrence technique. Precision Engineering 36(3), 458-466 (2012)

35. Engelbrecht, A.: Computational intelligence: an introduction. Wiley (2007)

36. Ferrari, S., Frosio, I., Piuri, V., Borghese, N.A.: Automatic multiscale meshing through HRBF networks. IEEE Trans. on Instrumentation and Measurement 54(4), 1463-1470 (2005)

37. Ferrari, S., Piuri, V.: Neural networks in intelligent sensors and measurement systems for industrial applications. In: S. Ablameyko, L. Goras, M. Gori, V. Piuri (eds.) Neural networks for instrumentation, measurement, and related industrial applications, pp. 19-42. IOS Press (2003)

38. Ferrari, S., Piuri, V., Scotti, F.: Image processing for granulometry analysis via neural networks. In: Proc. of the 2008 IEEE Int. Conf. on Computational Intelligence for Measurement Systems and Applications (CIMSA), pp. 28-32 (2008)

39. Foo, P.H., Ng, G.W.: High-level information fusion: An overview. Journal on Advances in Information Fusion 8(1), 33-72 (2013)

40. Fortuna, L., Giannone, P., Graziani, S., Xibilia, M.G.: Virtual instruments based on stacked neural networks to improve product quality monitoring in a refinery. IEEE Trans. on Instrumentation and Measurement 56(1), 95-101 (2007) 
41. Fortuna, L., Graziani, S., Xibilia, M.: Soft sensors for product quality monitoring in debutanizer distillation columns. Control Engineering Practice 13(4), 499-508 (2005)

42. Fowler, K.: Sensor survey part 2: Sensors and sensor networks in five years. IEEE Instrumentation Measurement Magazine 12(2), 40-44 (2009)

43. Fuente, M.J., Garcia-Alvarez, D., Sainz-Palmero, G.I., Vega, P.: Fault detection in a wastewater treatment plant based on neural networks and PCA. In: Proc. of the $201220^{\text {th }}$ Mediterranean Conf. on Control Automation (MED), pp. 758-763 (2012)

44. Gamassi, M., Piuri, V., Scotti, F., Roveri, M.: Genetic techniques for pattern extraction in particle boards images. In: Proc. of the 2006 IEEE Int. Conf. on Computational Intelligence for Measurement Systems and Applications (CIMSA), pp. 129-134 (2006)

45. García Nieto, P.J., Combarro, E.F., del Coz Díaz, J.J., Montañés, E.: A SVM-based regression model to study the air quality at local scale in Oviedo urban area (Northern Spain): A case study. Applied Mathematics and Computation 219(17), 8923-8937 (2013)

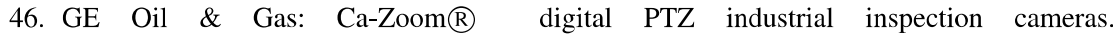
https://www.gemeasurement.com/inspection-ndt/remote-visual-inspection/ca-zoomindustrial-ptz-cameras

47. Genovese, A., Donida Labati, R., Piuri, V., Scotti, F.: Wildfire smoke detection using computational intelligence techniques. In: Proc. of the 2011 IEEE Int. Conf. on Computational Intelligence for Measurement Systems and Applications (CIMSA), pp. 1-6 (2011)

48. Gungor, V.C., Hancke, G.P.: Industrial wireless sensor networks: Challenges, design principles, and technical approaches. IEEE Trans. on Industrial Electronics 56(10), 4258-4265 (2009)

49. Haykin, S.: Neural networks and learning machines. v. 10. Prentice Hall (2009)

50. Hibert, C., Grandjean, G., Bitri, A., Travelletti, J., Malet, J.P.: Characterizing landslides through geophysical data fusion: Example of the La Valette landslide (France). Engineering Geology 128, 23-29 (2012)

51. Hossain, M., Rekabdar, B., Louis, S.J., Dascalu, S.: Forecasting the weather of nevada: A deep learning approach. In: Proc. of the 2015 Int. Joint Conf. on Neural Networks (IJCNN), pp. 1-6 (2015)

52. Hou, L., Bergmann, N.W.: Novel industrial wireless sensor networks for machine condition monitoring and fault diagnosis. IEEE Trans. on Instrumentation and Measurement 61(10), 2787-2798 (2012)

53. Ince, T., Kiranyaz, S., Eren, L., Askar, M., Gabbouj, M.: Real-time motor fault detection by 1-D convolutional neural networks. IEEE Trans. on Industrial Electronics 63(11), 7067-7075 (2016)

54. Jain, A.K., Duin, R.P.W., Mao, J.: Statistical pattern recognition: A review. IEEE Trans. on Pattern Analysis and Machine Intelligence 22(1), 4-37 (2000)

55. Karri, V., Ho, T., Madsen, O.: Artificial neural networks and neuro-fuzzy inference systems as virtual sensors for hydrogen safety prediction. Int. Journal of Hydrogen Energy 33(11), 2857-2867 (2008)

56. Khaleghi, B., Khamis, A., Karray, F.O., Razavi, S.N.: Multisensor data fusion: A review of the state-of-the-art. Information Fusion 14(1), 28-44 (2013)

57. Khan, S.A., Shahani, D.T., Agarwala, A.K.: Sensor calibration and compensation using artificial neural network. ISA Transactions 42(3), 337-352 (2003)

58. Kothari, V., Anuradha, J., Shah, S., Mittal, P.: A survey on particle swarm optimization in feature selection, pp. 192-201. Springer Berlin Heidelberg (2012)

59. Kreibich, O., Neuzil, J., Smid, R.: Quality-based multiple-sensor fusion in an industrial wireless sensor network for MCM. IEEE Trans. on Industrial Electronics 61(9), 4903-4911 (2014)

60. Krömer, P., Platoš, J., Snášel, V.: Mining multi-class industrial data with evolutionary fuzzy rules. In: Proc. of the 2013 IEEE Int. Conf. on Cybernetics (CYBCONF), pp. 191-196 (2013)

61. Kulkarni, R.V., Forster, A., Venayagamoorthy, G.K.: Computational intelligence in wireless sensor networks: A survey. IEEE Communications Surveys Tutorials 13(1), 68-96 (2011) 
62. Langone, R., Alzate, C., De Ketelaere, B., Vlasselaer, J., Meert, W., Suykens, J.A.K.: LSSVM based spectral clustering and regression for predicting maintenance of industrial machines. Engineering Applications of Artificial Intelligence 37, 268-278 (2015)

63. Larios, D.F., Barbancho, J., Rodríguez, G., Sevillano, J.L., Molina, F.J., León, C.: Energy efficient wireless sensor network communications based on computational intelligent data fusion for environmental monitoring. IET Communications 6(14), 2189 (2012)

64. Lecun, Y., Bengio, Y., Hinton, G.: Deep learning. Nature 521(7553), 436-444 (2015)

65. Lerner, B., Guterman, H., Aladjem, M., Dinstein, I.: A comparative study of neural network based feature extraction paradigms. Pattern Recognition Letters 20(1), 7-14 (1999)

66. Li, G., Rong, M., Wang, X., Li, X., Li, Y.: Partial discharge patterns recognition with deep Convolutional Neural Networks. In: Proc. of the 2016 Int. Conf. on Condition Monitoring and Diagnosis (CMD), pp. 324-327 (2016)

67. Li, P., Li, N., Cao, M.: Meteorology features extraction for transmission line icing process based on Kohonen Self-Organizing Maps. In: Proc. of the 2010 Int. Conf. on Computer Design and Applications (ICCDA), pp. 430-433 (2010)

68. Liu, Q., Jin, D., Shen, J., Fu, Z., Linge, N.: A WSN-based prediction model of microclimate in a greenhouse using extreme learning approaches. In: Proc. of the $201618^{\text {th }}$ Int. Conf. on Advanced Communication Technology (ICACT), pp. 1-2 (2016)

69. Longbotham, N., Pacifici, F., Glenn, T., Zare, A., Volpi, M., Tuia, D., Christophe, E., Michel, J., Inglada, J., Chanussot, J., Du, Q.: Multi-modal change detection, application to the detection of flooded areas: Outcome of the 2009-2010 data fusion contest. IEEE Journal of Selected Topics in Applied Earth Observations and Remote Sensing 5(1), 331-342 (2012)

70. Makhtar, A.K., Yussof, H., Al-Assadi, H., Yee, L.C., Othman, M.F., Shazali, K.: Wireless sensor network applications: A study in environment monitoring system. In: Proc. of the 2012 Int. Symp. on Robotics and Intelligent Sensors (IRIS), vol. 41, pp. 1204-1210 (2012)

71. Marco, S., Gutiérrez-Gálvez, A., Lansner, A., Martinez, D., Rospars, J.P., Beccherelli, R., Perera, A., Pearce, T.C., Verschure, P.F.M.J., Persaud, K.: A biomimetic approach to machine olfaction, featuring a very large-scale chemical sensor array and embedded neuro-bioinspired computation. Microsystem Technologies 20(4-5), 729-742 (2014)

72. Marwala, T.: Condition monitoring using computational intelligence methods: applications in mechanical and electrical systems. Springer Science \& Business Media (2012)

73. Marzano, F.S., Rivolta, G., Coppola, E., Tomassetti, B., Verdecchia, M.: Rainfall nowcasting from multisatellite passive-sensor images using a recurrent neural network. IEEE Trans. on Geoscience and Remote Sensing 45(11), 3800-3812 (2007)

74. Mohamed, R., Ahmed, A., Eid, A., Farag, A.: Support vector machines for camera calibration problem. In: Proc. of the 2006 Int. Conf. on Image Processing (ICIP), pp. 1029-1032 (2006)

75. Mourot, G., Bousghiri, S., Kratz, F.: Sensor fault detection using fuzzy logic and neural networks. In: Proc. of the Int. Conf. on Systems, Man and Cybernetics (SMC), pp. 369-374 (1993)

76. Muñoz, E., Ruspini, E.H.: Simulation of fuzzy queueing systems with a variable number of servers, arrival rate, and service rate. IEEE Trans. on Fuzzy Systems 22(4), 892-903 (2014)

77. Nakama, T., Muñoz, E., LeBlanc, K., Ruspini, E.: Generalizing and formalizing precisiation language to facilitate human-robot interaction. In: Computational Intelligence, pp. 381-397. Springer (2016)

78. Nor, A.S.M., Faramarzi, M., Yunus, M.A.M., Ibrahim, S.: Nitrate and sulfate estimations in water sources using a planar electromagnetic sensor array and artificial neural network method. IEEE Sensors Journal 15(1), 497-504 (2015)

79. O'Connor, E., Smeaton, A.F., O'Connor, N.E., Regan, F.: A neural network approach to smarter sensor networks for water quality monitoring. Sensors 12(4), 4605 (2012)

80. Palade, V., Bocaniala, C.D., Jain, L.: Computational intelligence in fault diagnosis. Springer (2006)

81. Paulinas, M., Ušinskas, A.: A survey of genetic algorithms applications for image enhancement and segmentation. Information Technology and control 36(3) (2015) 
82. phoneArena: A modern smartphone or a vintage supercomputer: which is more powerful? http://www.phonearena.com/news/A-modern-smartphone-or-a-vintage-supercomputerwhich-is-more-powerful_id57149 (2014)

83. Piuri, V., Scotti, F., Roveri, M.: Visual inspection of particle boards for quality assessment. In: Proc. of the IEEE Int. Conf. on Image Processing (ICIP), pp. 521-524 (2005)

84. Prieto, M.D., Cirrincione, G., Espinosa, A.G., Ortega, J.A., Henao, H.: Bearing fault detection by a novel condition-monitoring scheme based on statistical-time features and neural networks. IEEE Trans. on Industrial Electronics 60(8), 3398-3407 (2013)

85. Qiao, T., Ren, J., Craigie, C., Zabalza, J., Maltin, C., Marshall, S.: Quantitative prediction of beef quality using visible and NIR spectroscopy with large data samples under industry conditions. Journal of Applied Spectroscopy 82(1), 137-144 (2015)

86. Ribeiro, B.: Support vector machines for quality monitoring in a plastic injection molding process. IEEE Trans. on Systems, Man, and Cybernetics, Part C (Applications and Reviews) 35(3), 401-410 (2005)

87. Sagheer, A.: Piecewise one dimensional Self Organizing Map for fast feature extraction. In: Proc. of the $201010^{\text {th }}$ Int. Conf. on Intelligent Systems Design and Applications (ISDA), pp. 633-638 (2010)

88. Sammouda, R., Adgaba, N., Touir, A., Al-Ghamdi, A.: Agriculture satellite image segmentation using a modified artificial Hopfield neural network. Computers in Human Behavior 30, 436-441 (2014)

89. Sarcevic, P., Pletl, S., Kincses, Z.: Evolutionary algorithm based 9DOF sensor board calibration. In: Proc. of the $12^{\text {th }}$ IEEE Int. Symp. on Intelligent Systems and Informatics (SISY), pp. 187-192 (2014)

90. Shirvaikar, M.: Trends in automated visual inspection. Journal of Real-Time Image Processing 1(1), 41-43 (2006)

91. Simon, D.: Evolutionary Optimization Algorithms. Wiley (2013)

92. Singha, S., Bellerby, T.J., Trieschmann, O.: Satellite oil spill detection using Artificial Neural Networks. IEEE Journal of Selected Topics in Applied Earth Observations and Remote Sensing 6(6), 2355-2363 (2013)

93. Su, I.J., Tsai, C.C., Sung, W.T.: Area temperature system monitoring and computing based on adaptive fuzzy logic in wireless sensor networks. Applied Soft Computing 12(5), 1532-1541 (2012)

94. Szenkovits, A., Gaskó, N., Jahier, E.: Environment-model based testing with differential evolution in an industrial setting, pp. 819-830 (2016)

95. Teti, R., Segreto, T., Simeone, A., Teti, R.: Multiple sensor monitoring in nickel alloy turning for tool wear assessment via sensor fusion. In: Proc. of the $8^{\text {th }}$ CIRP Conf. on Intelligent Computation in Manufacturing Engineering, pp. 85-90 (2013)

96. Toh, K.K.V., Isa, N.A.M., Ashidi, N.: Noise adaptive fuzzy switching median filter for saltand-pepper noise reduction. IEEE Signal Processing Letters 17(3), 281-284 (2010)

97. Trillas, E., Eciolaza, L.: Fuzzy logic: an introductory course for engineering students. Springer Publishing Company, Incorporated (2015)

98. Vaseghi, S.V.: Advanced digital signal processing and noise reduction, $4^{\text {th }}$ edn. Wiley (2008)

99. Vos, T.E.J., Baars, A.I., Lindlar, F.F., Windisch, A., Wilmes, B., Gross, H., Kruse, P.M., Wegener, J.: Industrial case studies for evaluating search based structural testing. Int. Journal of Software Engineering and Knowledge Engineering 22(08), 1123-1149 (2012)

100. Vos, T.E.J., Lindlar, F.F., Wilmes, B., Windisch, A., Baars, A.I., Kruse, P.M., Gross, H., Wegener, J.: Evolutionary functional black-box testing in an industrial setting. Software Quality Journal 21(2), 259-288 (2013)

101. Wang, L., Fu, X.: Data mining with computational intelligence. Advanced information and knowledge processing. Springer, Berlin; New York (2005)

102. Wang, X.Y., Yang, H.Y., Zhang, Y., Fu, Z.K.: Image denoising using SVM classification in nonsubsampled contourlet transform domain. Information Sciences 246, 155-176 (2013)

103. Wijayasekara, D., Linda, O., Manic, M., Rieger, C.: FN-DFE: Fuzzy-neural data fusion engine for enhanced resilient state-awareness of hybrid energy systems. IEEE Trans. on Cybernetics 44(11), 2065-2075 (2014) 
104. Wu, J.L., Li, I.J.: A SOM-based dimensionality reduction method for KNN classifiers. In: Proc. of the 2010 Int. Conf. on System Science and Engineering (ICSSE), pp. 173-178 (2010)

105. Xiang, Y., Jiang, L.: Water quality prediction using LS-SVM and Particle Swarm Optimization. In: Proc. of the $2^{\text {nd }}$ Int. Workshop on Knowledge Discovery and Data Mining (WKDD), pp. 900-904 (2009)

106. Xie, X., Guo, J., Zhang, H., Jiang, T., Bie, R., Sun, Y.: Neural-network based structural health monitoring with wireless sensor networks. In: Proc. of the $20139^{\text {th }}$ Int. Conf. on Natural Computation (ICNC), pp. 163-167 (2013)

107. Xu, F., Song, X., Wang, X., Su, J.: Neural network model for earthquake prediction using DMETER data and seismic belt information. In: Proc. of the $20102^{\text {nd }}$ WRI Global Congress on Intelligent Systems (GCIS), vol. 3, pp. 180-183 (2010)

108. Xue, B., Zhang, M., Browne, W.N.: Particle swarm optimization for feature selection in classification: A multi-objective approach. IEEE Trans. on Cybernetics 43(6), 1656-1671 (2013)

109. Xue, B., Zhang, M., Browne, W.N., Yao, X.: A survey on evolutionary computation approaches to feature selection. IEEE Trans. on Evolutionary Computation 20(4), 606-626 (2016)

110. Yao, L., Lu, N., Jiang, S.: Artificial neural network (ANN) for multi-source PM2.5 estimation using surface, MODIS, and meteorological data. In: Proc. of the 2012 Int. Conf. on Biomedical Engineering and Biotechnology (iCBEB), pp. 1228-1231 (2012)

111. Ye, Y., Ci, S., Katsaggelos, A.K., Liu, Y., Qian, Y.: Wireless video surveillance: A survey. IEEE Access 1, 646-660 (2013)

112. Yildiz, A.R.: A new hybrid differential evolution algorithm for the selection of optimal machining parameters in milling operations. Applied Soft Computing 13(3), 1561-1566 (2013)

113. Yin, S., Ding, S.X., Xie, X., Luo, H.: A review on basic data-driven approaches for industrial process monitoring. IEEE Trans. on Industrial Electronics 61(11), 6418-6428 (2014)

114. Zhang, M., Liu, X.: A soft sensor based on adaptive fuzzy neural network and support vector regression for industrial melt index prediction. Chemometrics and Intelligent Laboratory Systems 126, 83-90 (2013)

115. Zhao, W., Du, S.: Spectral-spatial feature extraction for hyperspectral image classification: A dimension reduction and deep learning approach. IEEE Trans. on Geoscience and Remote Sensing 54(8), 4544-4554 (2016)

116. Zhao, Z., Liu, F.: Industrial monitoring based on moving average PCA and neural network. In: Proc. of the $30^{t h}$ Annual Conf. of IEEE Industrial Electronics Society (IECON), vol. 3, pp. 2168-2171 (2004) 